\title{
QUINTIC B-SPLINE COLLOCATION METHOD FOR NUMERICAL SOLUTION OF THE RLW EQUATION
}

\author{
BÜLENT SAKA ${ }^{凶 1}$, IDRIS DAĞ ${ }^{1}$ and DURSUN IRK ${ }^{1}$
}

(Received 10 March, 2007; revised 5 March, 2008)

\begin{abstract}
Quintic B-spline collocation schemes for numerical solution of the regularized long wave (RLW) equation have been proposed. The schemes are based on the CrankNicolson formulation for time integration and quintic B-spline functions for space integration. The quintic B-spline collocation method over finite intervals is also applied to the time-split RLW equation and space-split RLW equation. After stability analysis is applied to all the schemes, the results of the three algorithms are compared by studying the propagation of the solitary wave, interaction of two solitary waves and wave undulation.
\end{abstract}

2000 Mathematics subject classification: 65D07, 65N30, 65N35, 76B25.

Keywords and phrases: RLW equation, collocation, solitary waves, quintic B-splines, undular bore.

\section{Introduction}

The present study is concerned with numerical solution of the regularized long wave (RLW) equation, which was first derived by Peregrine to define undular bore development [15]. Since then, this equation has been used to model a large number of problems arising in various areas of applied science. The analytical solutions of the RLW equation together with some initial and boundary conditions are shown in $[3,5]$. Thus numerical solutions of this equation are of interest for various boundary and initial conditions. Well-known numerical techniques, including finite difference, finite element and Fourier pseudospectral methods, are applied to obtain numerical solutions of the RLW equation.

The B-spline functions are the basis for piecewise polynomials and are used to construct approximate solutions in the finite element techniques. So approximation solutions of the differential equations with B-splines can be obtained by the method of weighted residuals, of which the Galerkin and collocation methods are particular cases.

\footnotetext{
${ }^{1}$ Mathematics Department, Eskişehir Osmangazi University, 26480 Eskişehir, Turkey; e-mail: bsaka@ogu.edu.tr.

(C) Australian Mathematical Society 2008, Serial-fee code 0334-2700/08
} 
The Galerkin method is a widely used method for B-spline approximation. This method provides very smooth solutions for numerical solutions of partial differential equations. For example, Gardner et al. [11] proposed a Petrov-Galerkin quintic Bspline finite element method to obtain the numerical solution of the RLW equation. Burgers' equation is also numerically solved by the Galerkin method using quintic B-splines as both shape and weight functions over the finite elements [7]. The finite element solution using quadratic B-splines as the element and the weight functions for the space split EW equation is set up in [19]. But application of the Galerkin method accompanied by higher-degree polynomials results in a higher-degree matrix system. That brings a burden for numerical analysis, and the computational cost of the matrix system increases in the evaluation of both linear and nonlinear systems. On the other hand, the collocation method together with B-spline approximations represents an economical alternative since it only requires the evaluation of the unknown parameters at the grid points. A variant of this method has been successfully applied to solve differential equations [6, 8, 9, 12, 17, 20, 21, 23]. As is known, the success of the B-spline collocation method is dependent on the choice of Bspline basis. Quintic B-splines bases have been used to build up the approximation solutions for some nonlinear differential equations. For instance, a numerical solution of the Korteweg-de Vries (KdV) equation was obtained using collocation of quintic B-spline interpolation functions over finite elements in [10]. An algorithm based on the collocation method with quintic B-spline finite elements was set up to simulate the solutions of the KdV, Burgers' and KdVB equations [23].

The RLW equation has been used as a test equation for numerical methods since this equation can be solved analytically for some boundary and initial conditions. So a comparison between analytical and numerical solutions of the RLW equation can be carried out. Various forms of both B-spline collocation and B-spline Galerkin methods have been constructed in obtaining the numerical solutions of the RLW equation $[2,4,6,8,9,12,13,17,20,21]$. A numerical solution of the RLW equation has also been found by applying a splitting-up scheme, which is used to be able to construct the approximating solution with lower-degree piecewise polynomials, and both the applicability and the efficiency of the splitting of the differential equations are sought for numerical methods [6].

The organization of this paper is as follows. In Section 2.1, a quintic B-spline finite element algorithm is designed for the numerical solution of the RLW equation. In Section 2.2, the same method is applied to the time-split RLW equation. Lastly, in Section 2.3 the RLW equation is split into the first-order coupled matrix system by letting $V(x, t)=-U_{x}(x, t)$. The results of the three algorithms are compared in Section 3 using three test problems. The efficiencies of both time and space splitting techniques together with quintic B-splines are sought. In addition, some of the earlier results are also compared with those of the present algorithms.

The RLW equation is given by

$$
U_{t}+U_{x}+\varepsilon U U_{x}-\mu U_{x x t}=0
$$


where $\varepsilon$ and $\mu$ are positive parameters, and $x$ and $t$ denote differentiation. Boundary conditions are chosen from

$$
\begin{aligned}
U(a, t) & =\beta_{1}, \quad U(b, t)=\beta_{2}, \\
U_{x}(a, t) & =0, \quad U_{x}(b, t)=0,
\end{aligned}
$$

and the initial condition is

$$
U(x, 0)=f(x), \quad a \leq x \leq b,
$$

where $f(x)$ will be defined in the later sections depending on the test problems. The existence and uniqueness properties of the problem given by (1.1)-(1.3) were discussed by Bona and Bryant [5].

We consider a uniformly spatially distributed set of nodes

$$
a=x_{0}<x_{1}<\cdots<x_{N}=b
$$

over the solution domain $a \leq x \leq b$ with $h=x_{m}-x_{m-1}, m=1, \ldots, N$.

\section{Numerical method}

2.1. Quintic B-spline collocation method I (QBCM1) At the nodes $x_{m}$ the quintic B-splines $Q_{m}, m=-2, \ldots, N+2$, are defined by

$$
\begin{aligned}
& Q_{m}(x) \\
& \quad \frac{1}{h^{5}} \begin{cases}\left(x-x_{m-3}\right)^{5}, & {\left[x_{m-3}, x_{m-2}\right],} \\
\left(x-x_{m-3}\right)^{5}-6\left(x-x_{m-2}\right)^{5}, & {\left[x_{m-2}, x_{m-1}\right],} \\
\left(x-x_{m-3}\right)^{5}-6\left(x-x_{m-2}\right)^{5}+15\left(x-x_{m-1}\right)^{5}, & {\left[x_{m-1}, x_{m}\right],} \\
\left(x-x_{m-3}\right)^{5}-6\left(x-x_{m-2}\right)^{5}+15\left(x-x_{m-1}\right)^{5} & \\
-20\left(x-x_{m}\right)^{5}, & {\left[x_{m}, x_{m+1}\right],} \\
\left(x-x_{m-3}\right)^{5}-6\left(x-x_{m-2}\right)^{5}+15\left(x-x_{m-1}\right)^{5} & \\
-20\left(x-x_{m}\right)^{5}+15\left(x-x_{m+1}\right)^{5}, & {\left[x_{m+1}, x_{m+2}\right],} \\
-20\left(x-x_{m}\right)^{5}+15\left(x-x_{m+1}\right)^{5} & \\
-6\left(x-x_{m+2}\right)^{5}, & {\left[x_{m+2}, x_{m+3}\right],} \\
0, & \text { otherwise, }\end{cases}
\end{aligned}
$$

and the set of those B-splines defines the form of a basis over the interval $[a, b]$ (see [16]). A numerical solution of (1.1) will be derived by using the collocation method based on quintic B-splines. So a global approximation solution $U_{N}(x, t)$ to the analytical solution $U(x, t)$ will be sought in the form of an expansion of B-splines,

$$
U_{N}(x, t)=\sum_{m=-2}^{N+2} \delta_{m}(t) Q_{m}(x),
$$


where $Q_{m}$ are the quintic B-splines and $\delta_{m}$ are time-dependent parameters to be determined from the quintic B-spline collocation form of the RLW equation.

By using the approximation (2.2) and quintic B-splines (2.1), the nodal value $U$ and its first and second derivatives $U^{\prime}$ and $U^{\prime \prime}$ at the nodes $x_{i}$ are obtained in terms of the element parameters as

$$
\begin{aligned}
& U_{m}=U\left(x_{m}\right)=\delta_{m-2}+26 \delta_{m-1}+66 \delta_{m}+26 \delta_{m+1}+\delta_{m+2}, \\
& U_{m}^{\prime}=U^{\prime}\left(x_{m}\right)=\frac{5}{h}\left(\delta_{m+2}+10 \delta_{m+1}-10 \delta_{m-1}-\delta_{m-2}\right), \\
& U_{m}^{\prime \prime}=U^{\prime \prime}\left(x_{m}\right)=\frac{20}{h^{2}}\left(\delta_{m+2}+2 \delta_{m+1}-6 \delta_{m}+2 \delta_{m-1}+\delta_{m-2}\right),
\end{aligned}
$$

where ' and " denote first and second differentiation with respect to $x$ respectively.

Collocation points are selected to coincide with nodes. Substituting (2.3) into (1.1) leads to the set of the coupled first-order ordinary differential equations

$$
\begin{aligned}
& \AA_{m-2}+26 \AA_{m-1}+66 \AA_{m}+26 \AA_{m+1}+\AA_{m+2} \\
& \quad+\frac{5\left(1+\varepsilon z_{m}\right)}{h}\left(\delta_{m+2}+10 \delta_{m+1}-10 \delta_{m-1}-\delta_{m-2}\right) \\
& \quad-\frac{20 \mu}{h^{2}}\left(\AA_{m+2}+2 \AA_{m+1}-6 \AA_{m}+2 \AA_{m-1}+\AA_{m-2}\right)=0,
\end{aligned}
$$

where ${ }^{\circ}$ denotes derivative with respect to time and

$$
z_{m}=\delta_{m-2}+26 \delta_{m-1}+66 \delta_{m}+26 \delta_{m+1}+\delta_{m+2}
$$

Time discretization of (2.4) is carried out by interpolating time parameters $\delta_{m}$ and their time derivatives $\delta_{m}$ by using the Crank-Nicolson rule for $\delta_{m}$ and the usual forward difference rule for $\delta_{m}$ respectively between two time levels $n$ and $n+1$ :

$$
\delta_{m}=\frac{\delta_{m}^{n+1}+\delta_{m}^{n}}{2}, \quad \delta_{m}=\frac{\delta_{m}^{n+1}-\delta_{m}^{n}}{\Delta t}
$$

Thus a recurrence relationship is obtained between two successive time levels $n$ and $n+1$ which relates two successive unknown parameters $\delta_{i}^{n+1}$ and $\delta_{i}^{n}$ (where $i=m-2, \ldots, m+2)$ as

$$
\begin{aligned}
& \alpha_{m 1} \delta_{m-2}^{n+1}+\alpha_{m 2} \delta_{m-1}^{n+1}+\alpha_{m 3} \delta_{m}^{n+1}+\alpha_{m 4} \delta_{m+1}^{n+1}+\alpha_{m 5} \delta_{m+2}^{n+1} \\
& =\alpha_{m 5} \delta_{m-2}^{n}+\alpha_{m 4} \delta_{m-1}^{n}+\alpha_{m 3} \delta_{m}^{n}+\alpha_{m 2} \delta_{m+1}^{n}+\alpha_{m 1} \delta_{m+2}^{n}
\end{aligned}
$$


where

$$
\begin{aligned}
& \alpha_{m 1}=2 h^{2}-5 h \Delta t\left(1+\varepsilon z_{m}\right)-40 \mu, \\
& \alpha_{m 2}=52 h^{2}-50 h \Delta t\left(1+\varepsilon z_{m}\right)-80 \mu, \\
& \alpha_{m 3}=132 h^{2}+240 \mu, \\
& \alpha_{m 4}=52 h^{2}+50 h \Delta t\left(1+\varepsilon z_{m}\right)-80 \mu, \\
& \alpha_{m 5}=2 h^{2}+5 h \Delta t\left(1+\varepsilon z_{m}\right)-40 \mu, \quad m=0,1, \ldots, N .
\end{aligned}
$$

The above system consists of the $N+1$ equations in the $N+5$ unknown parameters. The elimination of parameters $\delta_{-2}^{n+1}, \quad \delta_{-1}^{n+1}, \delta_{N+1}^{n+1}, \delta_{N+2}^{n+1}$ from the system (2.6) using the boundary conditions $U(a, t)=\beta_{1}, U(b, t)=\beta_{2}$ and $U_{x}(a, t)=U_{x}(b, t)=0$ enables one to get a solvable $(N+1) \times(N+1)$ matrix system. The resulting pentadiagonal matrix system is easily and efficiently solved with a variant of the Thomas algorithms [18].

To initiate element parameters $\delta_{m}^{n}$, we must find the initial unknown parameters $\delta_{m}^{0}$ by means of the following requirements:

$$
\begin{aligned}
\left(U_{N}\right)_{x}(a, 0) & =\delta_{-2}^{0}+10 \delta_{-1}^{0}-10 \delta_{1}^{0}-\delta_{2}^{0}=0 \\
\left(U_{N}\right)_{x x}(a, 0) & =\delta_{-2}^{0}+2 \delta_{-1}^{0}-6 \delta_{0}^{0}+2 \delta_{1}^{0}+\delta_{2}^{0}=0 \\
U_{N}(x, 0) & =\delta_{m-2}^{0}+26 \delta_{m-1}^{0}+66 \delta_{m}^{0}+26 \delta_{m+1}^{0}+\delta_{m+2}^{0}=U\left(x_{m}, 0\right), \\
\left(U_{N}\right)_{x}(b, 0) & =\delta_{N+2}^{0}+10 \delta_{N+1}^{0}-10 \delta_{N-1}^{0}-\delta_{N-2}^{0}=0 \\
\left(U_{N}\right)_{x x}(b, 0) & =\delta_{N+2}^{0}+2 \delta_{N+1}^{0}-6 \delta_{N}^{0}+2 \delta_{N-1}^{0}+\delta_{N-2}^{0}=0 .
\end{aligned}
$$

This also allows us to determine the solution of the pentadiagonal matrix equation with the parameters $\delta_{m}^{0}, m=-2, \ldots, N+2$.

To deal with nonlinearity in (2.6), the time parameters $\delta_{m}$ in $z_{m}$ are replaced by $\delta_{m}^{n}$ for the time level, so the linearized algebraic system (2.6) is used together with the following iteration process to get a better result:

(1) calculate $\delta^{n+1}$ using iteration procedure (2.6) by employing the Thomas algorithms; and

(2) obtain a new approximation to $\left(\delta^{*}\right)^{n+1}$ by the procedure

$$
\left(\boldsymbol{\delta}^{*}\right)^{n+1}=\boldsymbol{\delta}^{n}+\frac{1}{2}\left(\boldsymbol{\delta}^{n+1}-\boldsymbol{\delta}^{n}\right) .
$$

Before moving the calculation of the next time step approximation for the time parameters, iteration should be repeated two or three times.

To investigate the stability of the difference scheme (2.6), we apply von Neumann stability analysis. We assume that the quantity $U$ in the nonlinear term $U U_{x}$ is locally constant $p$ for the RLW equation. This selection is the same as assuming that the corresponding values of $z_{m}$ are also constant and equal to $p$. Let us consider a particular solution, $\delta_{m}^{n}=q^{n} e^{i m \varphi}$, where $i$ is an imaginary unit, $\varphi$ is an arbitrary real number, and $q=q(\varphi)$ is a complex number whose value must be found. 
After substituting the Fourier mode $\delta_{m}^{n}=q^{n} e^{i m \varphi}$ into the linearized form of the difference equation, we have the growth factor

$$
q=\frac{a+i b}{a-i b}
$$

where

$$
\begin{aligned}
& a=\left(\alpha_{m 1}+\alpha_{m 5}\right) \cos 2 \varphi+\left(\alpha_{m 2}+\alpha_{m 4}\right) \cos \varphi+\alpha_{m 3}, \\
& b=\left(\alpha_{m 1}-\alpha_{m 5}\right) \sin 2 \varphi+\left(\alpha_{m 2}-\alpha_{m 4}\right) \sin \varphi
\end{aligned}
$$

Since the magnitude of the growth factor is $|q|=1$, the difference scheme (2.6) is unconditionally stable.

2.2. Quintic B-spline collocation method II (QBCM2) The time-split RLW equation is

$$
\begin{aligned}
\left(U-\mu U_{x x}\right)_{t}+2 \varepsilon U U_{x} & =0 \\
\left(U-\mu U_{x x}\right)_{t}+2 U_{x} & =0 .
\end{aligned}
$$

If we identify the collocation points with nodes $x_{m}$ and substitute the nodal values $U_{m}$ and their first two successive derivatives $U_{m}^{\prime}$ and $U_{m}^{\prime \prime}$ into (2.10) and (2.11), we have the following coupled matrix system of first-order ordinary differential equations:

$$
\begin{aligned}
& \AA_{m-2}+26 \AA_{m-1}+66 \AA_{m}+26 \AA_{m+1}+\AA_{m+2} \\
& -\frac{20 \mu}{h^{2}}\left(\AA_{m+2}+2 \AA_{m+1}-6 \AA_{m}+2 \AA_{m-1}+\AA_{m-2}\right) \\
& \quad+\frac{10 \varepsilon z_{m}}{h}\left(\delta_{m+2}+10 \delta_{m+1}-10 \delta_{m-1}-\delta_{m-2}\right)=0, \\
& \AA_{m-2}+26 \AA_{m-1}+66 \AA_{m}+26 \AA_{m+1}+\AA_{m+2} \\
& \quad-\frac{20 \mu}{h^{2}}\left(\AA_{m+2}+2 \AA_{m+1}-6 \AA_{m}+2 \delta_{m-1}+\AA_{m-2}\right) \\
& \quad+\frac{10}{h}\left(\delta_{m+2}+10 \delta_{m+1}-10 \delta_{m-1}-\delta_{m-2}\right)=0,
\end{aligned}
$$

where again ${ }^{\circ}$ denotes derivative with respect to time and

$$
z_{m}=\delta_{m-2}+26 \delta_{m-1}+66 \delta_{m}+26 \delta_{m+1}+\delta_{m+2}
$$

With a Crank-Nicolson formulation in time for the unknown parameters $\delta_{m}$ and the usual finite difference scheme for their time derivatives $\AA_{m}$,

$$
\delta_{m}=\frac{\delta_{m}^{n}+\delta_{m}^{n+1 / 2}}{2}, \quad \AA_{m}=\frac{\delta_{m}^{n+1 / 2}-\delta_{m}^{n}}{\Delta t},
$$


we obtain an equation between two time levels, for the unknown parameters of (2.12) relating time levels $n$ and $n+1 / 2, \delta_{i}^{n}$ to $\delta_{i}^{n+1 / 2}(i=m-2, \ldots, m+2)$,

$$
\begin{gathered}
\alpha_{1} \delta_{m-2}^{n+1 / 2}+\alpha_{2} \delta_{m-1}^{n+1 / 2}+\alpha_{3} \delta_{m}^{n+1 / 2}+\alpha_{4} \delta_{m+1}^{n+1 / 2}+\alpha_{5} \delta_{m+2}^{n+1 / 2} \\
=\alpha_{5} \delta_{m-2}^{n}+\alpha_{4} \delta_{m-1}^{n}+\alpha_{3} \delta_{m}^{n}+\alpha_{2} \delta_{m+1}^{n}+\alpha_{1} \delta_{m+2}^{n}
\end{gathered}
$$

where

$$
\begin{aligned}
& \alpha_{1}=2 h^{2}-5 h \Delta t \varepsilon z_{m}-40 \mu, \\
& \alpha_{2}=52 h^{2}-50 h \Delta t \varepsilon z_{m}-80 \mu, \\
& \alpha_{3}=132 h^{2}+240 \mu, \\
& \alpha_{4}=52 h^{2}+50 h \Delta t \varepsilon z_{m}-80 \mu, \\
& \alpha_{5}=2 h^{2}+5 h \Delta t \varepsilon z_{m}-40 \mu .
\end{aligned}
$$

Similarly, using the Crank-Nicolson approach for parameters $\delta_{m}$ and the finite difference scheme for their time derivatives $\AA_{m}$ respectively between two time levels $n+1 / 2$ and $n+1$ in (2.13),

$$
\delta_{m}=\frac{\delta_{m}^{n+1}+\delta_{m}^{n+1 / 2}}{2}, \quad \AA_{m}=\frac{\delta_{m}^{n+1}-\delta_{m}^{n+1 / 2}}{\Delta t},
$$

we have an equation relating $\delta_{i}^{n+1 / 2}$ to $\delta_{i}^{n+1}(i=m-2, \ldots, m+2)$,

$$
\begin{aligned}
& \alpha_{6} \delta_{m-2}^{n+1}+\alpha_{7} \delta_{m-1}^{n+1}+\alpha_{8} \delta_{m}^{n+1}+\alpha_{9} \delta_{m+1}^{n+1}+\alpha_{10} \delta_{m+2}^{n+1} \\
& \quad=\alpha_{10} \delta_{m-2}^{n+1 / 2}+\alpha_{9} \delta_{m-1}^{n+1 / 2}+\alpha_{8} \delta_{m}^{n+1 / 2}+\alpha_{7} \delta_{m+1}^{n+1 / 2}+\alpha_{6} \delta_{m+2}^{n+1 / 2}
\end{aligned}
$$

where

$$
\begin{aligned}
\alpha_{6} & =2 h^{2}-5 h \Delta t-40 \mu, \\
\alpha_{7} & =52 h^{2}-50 h \Delta t-80 \mu, \\
\alpha_{8} & =132 h^{2}+240 \mu, \\
\alpha_{9} & =52 h^{2}+50 h \Delta t-80 \mu, \\
\alpha_{10} & =2 h^{2}+5 h \Delta t-40 \mu .
\end{aligned}
$$

Equations (2.15) and (2.17) constitute a multistep finite difference scheme, for solving the RLW equation, having $N+1$ equations containing $N+5$ unknown parameters. We obtain a unique solution by eliminating the parameters $\delta_{-2}^{i}$, $\delta_{-1}^{i}, \delta_{N+1}^{i}, \delta_{N+2}^{i}(i=n+1 / 2, n+1)$ from (2.15) and (2.17) after imposition of the boundary conditions $U(a, t)=\beta_{1}, U(b, t)=\beta_{2}$ and $U_{x}(a, t)=U_{x}(b, t)=0$. Boundary conditions $U(a, t)=\beta_{1}$ and $U_{x}(a, t)=0$ are used to eliminate $\delta_{-2}^{i}$ and $\delta_{-1}^{i}$, while the conditions $U(b, t)=\beta_{1}$ and $U_{x}(b, t)=0$ are used to eliminate $\delta_{N+1}^{i}$ 
and $\delta_{N+2}^{i}$, so that this solvable five-banded pentadiagonal matrix system is solved by way of the Thomas algorithms. Once we find an approximation $\delta_{m}^{0}$, the next time solution parameters $\delta_{m}^{n+1}$ having been found, parameters $\delta_{m}^{n+1 / 2}$ from (2.15) are computed using (2.17).

To handle the nonlinearity in (2.15), the corrector procedure

$$
\left(\boldsymbol{\delta}^{*}\right)^{n+1 / 2}=\boldsymbol{\delta}^{n}+\frac{1}{2}\left(\boldsymbol{\delta}^{n+1 / 2}-\boldsymbol{\delta}^{n}\right)
$$

is used. To start the iteration for calculating the unknown parameters, the initial unknown parameters $\delta_{m}^{0}$ must be determined from the initial and boundary conditions to satisfy the requirements given in (2.8).

To investigate the stability of the difference equation (2.15), we apply the von Neumann stability method after linearizing by taking $U$ in the nonlinear term $U U_{x}$ as a local constant $p$. Thus the term $z_{m}$ in the difference equation corresponds to constant $p$. Substitute $\delta_{m}^{n}=q^{n} e^{i m \varphi}$ in (2.15), then

$$
q=\frac{a+i b}{a-i b}
$$

where the quantities $q, \varphi$ have the same meaning as in previous considerations and

$$
\begin{aligned}
& a=\left(\alpha_{1}+\alpha_{5}\right) \cos 2 \varphi+\left(\alpha_{2}+\alpha_{4}\right) \cos \varphi+\alpha_{3}, \\
& b=\left(\alpha_{1}-\alpha_{5}\right) \sin 2 \varphi+\left(\alpha_{2}-\alpha_{4}\right) \sin \varphi .
\end{aligned}
$$

Von Neumann's condition $|q| \leq 1$ is satisfied so that the difference scheme is unconditionally stable. With the similar calculation the difference equation (2.17) is also unconditionally stable.

2.3. Quintic B-spline collocation method III (QBCM3) In this section, the quintic B-spline collocation method discussed above is employed to obtain the numerical solution of the space-split RLW equation. If we set $V(x, t)=-U_{x}(x, t)$ in the RLW equation, it becomes

$$
\begin{gathered}
U_{t}-(1+\varepsilon U) V+\mu V_{x t}=0, \\
V+U_{x}=0 .
\end{gathered}
$$

The boundary and initial conditions can be rewritten as

$$
\begin{gathered}
U(a, t)=\beta_{1}, \quad U(b, t)=\beta_{2}, \quad V(a, t)=0, \quad V(b, t)=0, \\
U_{x}(a, t)=0, \quad U_{x}(b, t)=0, \quad V_{x}(a, t)=0, \quad V_{x}(b, t)=0, \\
U(x, 0)=f(x), \quad V(x, 0)=-f^{\prime}(x), \quad a \leq x \leq b .
\end{gathered}
$$

Expressing $U(x, t)$ and $V(x, t)$ by using quintic B-splines, $Q_{m}(x)$ and the timedependent parameters $\delta_{m}$ and $\sigma_{m}$ are given as

$$
U_{N}(x, t)=\sum_{m=-2}^{N+2} \delta_{m}(t) Q_{m}(x), \quad V_{N}(x, t)=\sum_{m=-2}^{N+2} \sigma_{m}(t) Q_{m}(x) .
$$


The nodal variables $U_{m}, V_{m}$ and their space derivatives $U_{m}^{\prime}, V_{m}^{\prime}$ can be calculated by using expressions (2.22) and the quintic B-splines (2.1) as

$$
\begin{aligned}
U_{m} & =U\left(x_{m}\right)=\delta_{m-2}+26 \delta_{m-1}+66 \delta_{m}+26 \delta_{m+1}+\delta_{m+2}, \\
U_{m}^{\prime} & =U^{\prime}\left(x_{m}\right)=\frac{5}{h}\left(\delta_{m+2}+10 \delta_{m+1}-10 \delta_{m-1}-\delta_{m-2}\right), \\
V_{m} & =V\left(x_{m}\right)=\sigma_{m-2}+26 \sigma_{m-1}+66 \sigma_{m}+26 \sigma_{m+1}+\sigma_{m+2}, \\
V_{m}^{\prime} & =V^{\prime}\left(x_{m}\right)=\frac{5}{h}\left(\sigma_{m+2}+10 \sigma_{m+1}-10 \sigma_{m-1}-\sigma_{m-2}\right) .
\end{aligned}
$$

Substituting (2.23) into (2.19) yields

$$
\begin{aligned}
& \delta_{m-2}+26 \AA_{m-1}+66 \AA_{m}+26 \AA_{m+1}+\AA_{m+2} \\
&-\left(1+\varepsilon z_{m}\right)\left(\sigma_{m-2}+26 \sigma_{m-1}+66 \sigma_{m}+26 \sigma_{m+1}+\sigma_{m+2}\right) \\
&+ \frac{5 \mu}{h}\left(\stackrel{\circ}{\sigma}_{m+2}+10 \circ_{m+1}-10 \circ_{m-1}-\stackrel{\circ}{\sigma}_{m-2}\right) \\
& \quad= 0 \\
& \sigma_{m-2}+26 \sigma_{m-1}+66 \sigma_{m}+26 \sigma_{m+1}+\sigma_{m+2} \\
& \quad+\frac{5}{h}\left(\delta_{m+2}+10 \delta_{m+1}-10 \delta_{m-1}-\delta_{m-2}\right) \\
&=0
\end{aligned}
$$

where ${ }^{\circ}$ denotes differentiation with respect to time and

$$
z_{m}=\delta_{m-2}+26 \delta_{m-1}+66 \delta_{m}+26 \delta_{m+1}+\delta_{m+2} .
$$

To derive the fully discretized difference equation, we use the following CrankNicolson approximation in time for the unknown parameters $\delta_{m}, \sigma_{m}$ and the usual finite difference scheme for the time derivative of the parameters $\AA_{m}, \stackrel{\circ}{\sigma}_{m}$ :

$$
\begin{aligned}
& \delta_{m}=\frac{\delta_{m}^{n+1}+\delta_{m}^{n}}{2}, \quad \delta_{m}=\frac{\delta_{m}^{n+1}-\delta_{m}^{n}}{\Delta t}, \\
& \sigma_{m}=\frac{\sigma_{m}^{n+1}+\sigma_{m}^{n}}{2}, \stackrel{\circ}{\sigma}_{m}=\frac{\sigma_{m}^{n+1}-\sigma_{m}^{n}}{\Delta t} .
\end{aligned}
$$

These lead to a system of $2 N+2$ algebraic equations in $2 N+10$ unknowns:

$$
\begin{aligned}
& 2 h \delta_{m-2}^{n+1}+\beta_{m 1} \sigma_{m-2}^{n+1}+52 h \delta_{m-1}^{n+1}+\beta_{m 2} \sigma_{m-1}^{n+1}+132 h \delta_{m}^{n+1} \\
& +\beta_{m 3} \sigma_{m}^{n+1}+52 h \delta_{m+1}^{n+1}+\beta_{m 4} \sigma_{m+1}^{n+1}+2 h \delta_{m+2}^{n+1}+\beta_{m 5} \sigma_{m+2}^{n+1} \\
& =2 h \delta_{m-2}^{n+}-\beta_{m 5} \sigma_{m-2}^{n}+52 h \delta_{m-1}^{n}-\beta_{m 4} \sigma_{m-1}^{n}+132 h \delta_{m}^{n}-\beta_{m 3} \sigma_{m}^{n} \\
& \quad+52 h \delta_{m+1}^{n}-\beta_{m 2} \sigma_{m+1}^{n}+2 h \delta_{m+2}^{n}-\beta_{m 1} \sigma_{m+2}^{n}
\end{aligned}
$$




$$
\begin{aligned}
& -5 \delta_{m-2}^{n+1}+h \sigma_{m-2}^{n+1}-50 \delta_{m-1}^{n+1}+26 h \sigma_{m-1}^{n+1}+66 h \sigma_{m}^{n+1} \\
& +50 \delta_{m+1}^{n+1}+26 h \sigma_{m+1}^{n+1}+5 \delta_{m+2}^{n+1}+h \sigma_{m+2}^{n+1} \\
& \quad=5 \delta_{m-2}^{n+h}-h \sigma_{m-2}^{n}+50 \delta_{m-1}^{n}-26 h \sigma_{m-1}^{n}-66 h \sigma_{m}^{n} \\
& \quad \quad-50 \delta_{m+1}^{n}-26 h \sigma_{m+1}^{n}-5 \delta_{m+2}^{n}-h \sigma_{m+2}^{n}, \quad m=0, \ldots, N
\end{aligned}
$$

where

$$
\begin{array}{llrl}
\beta_{m 1}=-d h \Delta t-10 \mu, & \beta_{m 2}=-26 d h \Delta t-100 \mu, & \beta_{m 3}=-66 d h \Delta t, \\
\beta_{m 4}=-26 d h \Delta t+100 \mu, & \beta_{m 5}=-d h \Delta t+10 \mu, & d=1+\varepsilon z_{m} .
\end{array}
$$

Application of the boundary conditions

$$
\begin{gathered}
U(a, t)=\beta_{1}, \quad U_{x}(a, t)=0, \quad V(a, t)=0, \quad V_{x}(a, t)=0, \\
U(b, t)=\beta_{2}, \quad U_{x}(b, t)=0, \quad V(b, t)=0 \quad \text { and } \quad V_{x}(b, t)=0
\end{gathered}
$$

enables us to eliminate the parameters $\delta_{-2}^{n+1}, \sigma_{-2}^{n+1}, \delta_{-1}^{n+1}, \sigma_{-1}^{n+1}, \delta_{N+1}^{n+1}, \sigma_{N+1}^{n+1}, \delta_{N+2}^{n+1}$ and $\sigma_{N+2}^{n+1}$ from the system (2.26) so that we have a solvable 11-banded matrix system of dimension $(2 N+2) \times(2 N+2)$. This system is solved by employing the Gauss elimination procedure.

Time evolution of the parameters $\delta_{m}^{n+1}, \sigma_{m}^{n+1}$ is computed once the initial parameters $\delta_{m}^{0}, \sigma_{m}^{0}$ are obtained with the help of the following boundary and initial conditions:

$$
\begin{aligned}
\left(U_{N}\right)_{x}(a, 0) & =\delta_{-2}^{0}+10 \delta_{-1}^{0}-10 \delta_{1}^{0}-\delta_{2}^{0}=0 \\
\left(U_{N}\right)_{x x}(a, 0) & =\delta_{-2}^{0}+2 \delta_{-1}^{0}-6 \delta_{0}^{0}+2 \delta_{1}^{0}+\delta_{2}^{0}=0 \\
U_{N}(x, 0) & =\delta_{m-2}^{0}+26 \delta_{m-1}^{0}+66 \delta_{m}^{0}+26 \delta_{m+1}^{0}+\delta_{m+2}^{0}=U\left(x_{m}, 0\right), \\
\left(U_{N}\right)_{x}(b, 0) & =\delta_{N+2}^{0}+10 \delta_{N+1}^{0}-10 \delta_{N-1}^{0}-\delta_{N-2}^{0}=0 \\
\left(U_{N}\right)_{x x}(b, 0) & =\delta_{N+2}^{0}+2 \delta_{N+1}^{0}-6 \delta_{N}^{0}+2 \delta_{N-1}^{0}+\delta_{N-2}^{0}=0, \\
\left(V_{N}\right)_{x}(a, 0) & =\sigma_{-2}^{0}+10 \sigma_{-1}^{0}-10 \sigma_{1}^{0}-\sigma_{2}^{0}=0 \\
\left(V_{N}\right)_{x x}(a, 0) & =\sigma_{-2}^{0}+2 \sigma_{-1}^{0}-6 \sigma_{0}^{0}+2 \sigma_{1}^{0}+\sigma_{2}^{0}=0, \\
V_{N}(x, 0) & =\sigma_{m-2}^{0}+26 \sigma_{m-1}^{0}+66 \sigma_{m}^{0}+26 \sigma_{m+1}^{0}+\sigma_{m+2}^{0}=V\left(x_{m}, 0\right), \\
\left(V_{N}\right)_{x}(b, 0) & =\sigma_{N+2}^{0}+10 \sigma_{N+1}^{0}-10 \sigma_{N-1}^{0}-\sigma_{N-2}^{0}=0, \\
\left(V_{N}\right)_{x x}(b, 0) & =\sigma_{N+2}^{0}+2 \sigma_{N+1}^{0}-6 \sigma_{N}^{0}+2 \sigma_{N-1}^{0}+\sigma_{N-2}^{0}=0 .
\end{aligned}
$$

The system above is also solved by a variant of the Thomas algorithms.

The procedure defined in (2.9) is applied two or three times at every time step to accomplish better results.

To investigate the stability of the difference scheme (2.26), we apply the method of harmonics [22] after linearizing by taking $U$ in the nonlinear term $U V$ as a local 
constant $p$. Thus the term $z_{m}$ in the difference equation corresponds to a constant $p$. In this case, we will try to find the solution in the form

$$
\begin{aligned}
\delta_{m}^{n} & =P q^{n} e^{i m \varphi}, \\
\sigma_{m}^{n} & =W q^{n} e^{i m \varphi},
\end{aligned}
$$

where the quantities $q$ and $\varphi$ have the same meaning as in the previous two considerations and $P, W$ are the amplitudes of harmonics. Substituting $\delta_{m}^{n}=P q^{n} e^{i m \varphi}$ and $\sigma_{m}^{n}=W q^{n} e^{i m \varphi}$ into the difference scheme (2.26) gives

$$
\begin{array}{r}
a_{1} P+\left(b_{1}+i c_{1}\right) W=0, \\
i a_{2} P+b_{2} W=0,
\end{array}
$$

where

$$
\begin{aligned}
& a_{1}=(2 h \cos 2 \varphi+52 h \cos \varphi+132 h+52 h \cos \varphi+2 h \cos 2 \varphi)(q-1), \\
& b_{1}=\left(\beta_{1} \cos 2 \varphi+\beta_{2} \cos \varphi+\beta_{3}+\beta_{4} \cos \varphi+\beta_{5} \cos 2 \varphi\right)(q+1), \\
& c_{1}=\left(-\beta_{1} \sin 2 \varphi-\beta_{2} \sin \varphi+\beta_{4} \sin \varphi+\beta_{5} \sin 2 \varphi\right)(q-1), \\
& a_{2}=(10 \sin 2 \varphi+100 \sin \varphi)(q+1), \\
& b_{2}=(h \cos 2 \varphi+26 h \cos \varphi+66 h+26 h \cos \varphi+h \cos 2 \varphi)(q+1) .
\end{aligned}
$$

The system (2.28), with respect to $P$ and $W$, will have a nontrivial solution if the determinant of the coefficient matrix is equal to zero. After some mathematical manipulation, the roots of the determinant of the coefficient matrix can be found as

$$
q_{1}=\frac{z_{1}}{z_{2}}, \quad q_{2}=-1
$$

where

$$
\begin{aligned}
z_{1}= & -90 d h \Delta t \sin ^{3} \varphi+490 d h \Delta t \sin \varphi+410 d h \Delta t \cos \varphi \sin \varphi \\
& -5 d h \Delta t \cos \varphi \sin ^{3} \varphi+i\left(-406 h^{2} \sin ^{2} \varphi+1300 \mu \sin ^{2} \varphi+916 h^{2}\right. \\
& +500 \mu \sin ^{2} \varphi \cos \varphi-50 \mu \sin ^{4} \varphi+2 h^{2} \sin ^{4} \varphi+884 h^{2} \cos \varphi \\
& \left.-52 h^{2} \sin ^{2} \varphi \cos \varphi\right), \\
z_{2}= & 90 d h \Delta t \sin ^{3} \varphi-490 d h \Delta t \sin \varphi-410 d h \Delta t \cos \varphi \sin \varphi \\
& +5 d h \Delta t \cos \varphi \sin ^{3} \varphi+i\left(-406 h^{2} \sin ^{2} \varphi+1300 \mu \sin ^{2} \varphi+916 h^{2}\right. \\
& +500 \mu \sin ^{2} \varphi \cos \varphi-50 \mu \sin ^{4} \varphi+2 h^{2} \sin ^{4} \varphi+884 h^{2} \cos \varphi \\
& \left.-52 h^{2} \sin ^{2} \varphi \cos \varphi\right) .
\end{aligned}
$$

Since $\left|q_{1}\right| \leq 1$ and $\left|q_{2}\right| \leq 1$ are satisfied, the difference scheme is unconditionally stable. 


\section{Numerical calculations}

The only three conservation quantities of the RLW equation are [14]

$$
\begin{aligned}
& C_{1}=\int_{a}^{b} U d x \simeq h \sum_{j=1}^{N} U_{j}^{n} \\
& C_{2}=\int_{a}^{b}\left(U^{2}+\mu\left(U_{x}\right)^{2}\right) d x \simeq h \sum_{j=1}^{N}\left\{\left(U_{j}^{n}\right)^{2}+\mu\left(\left(U_{x}\right)_{j}^{n}\right)^{2}\right\} \\
& C_{3}=\int_{a}^{b}\left(U^{3}+3 U^{2}\right) d x \simeq h \sum_{j=1}^{N}\left\{\left(U_{j}^{n}\right)^{3}+3\left(U_{j}^{n}\right)^{2}\right\}
\end{aligned}
$$

The integrals above are approximated by the rectangle rule for quadrature. Thus $U_{j}^{n}$ and its first derivative are calculated from (2.3). Conservation quantities, the $L_{2}$ error norm

$$
L_{2}=\sqrt{h \sum_{j=0}^{N}\left|U_{j}^{\text {exact }}-\left(U_{N}\right)_{j}\right|^{2}}
$$

and the $L_{\infty}$ error norm

$$
L_{\infty}=\left\|U^{\text {exact }}-U_{N}\right\|_{\infty}=\max _{j}\left|U_{j}^{\text {exact }}-\left(U_{N}\right)_{j}\right|
$$

will be computed to show how well the behaviour of the numerical schemes models the test problems in terms of accuracy.

3.1. Single solitary wave The exact solution of (1.1) is given by

$$
U(x, t)=3 c \operatorname{sech}^{2}\left(k\left[x-x_{0}-v t\right]\right),
$$

which represents a single solitary wave of amplitude $3 c$, velocity $v=1+\varepsilon c$ and $k=\frac{1}{2}(\varepsilon c / \mu \nu)^{1 / 2}$ (see [15]). This solution travels across the interval $-40 \leq x \leq 60$ in the time period $0 \leq t \leq 20$ with parameters $\varepsilon=\mu=1, x_{0}=0$ and boundary conditions $\beta_{1}=0, \beta_{2}=0$.

The numerical experiment of this single solitary wave motion is performed for the three schemes. The experiment is carried out using the initial condition

$$
U(x, 0)=3 c \operatorname{sech}^{2}\left(k\left[x-x_{0}\right]\right) .
$$

First, the program is run up to time $t=20$ with parameters $c=0.1, \Delta t=0.1$ and $h=0.125$, which are chosen to be the same as in some earlier papers $[6,11,12$, $20,21]$. The results of the error norms and conservation invariants are documented in Table 1 at times $t=0$ and 20 for the three schemes. During the program run, numerical invariants $C_{2}$ and $C_{3}$ remain constant and $C_{1}$ remains almost constant. Although the three schemes produce the same order accuracy, QBCM1 and QBCM3 give a slightly smaller error than QBCM2. The initial function and numerical solution are drawn for 


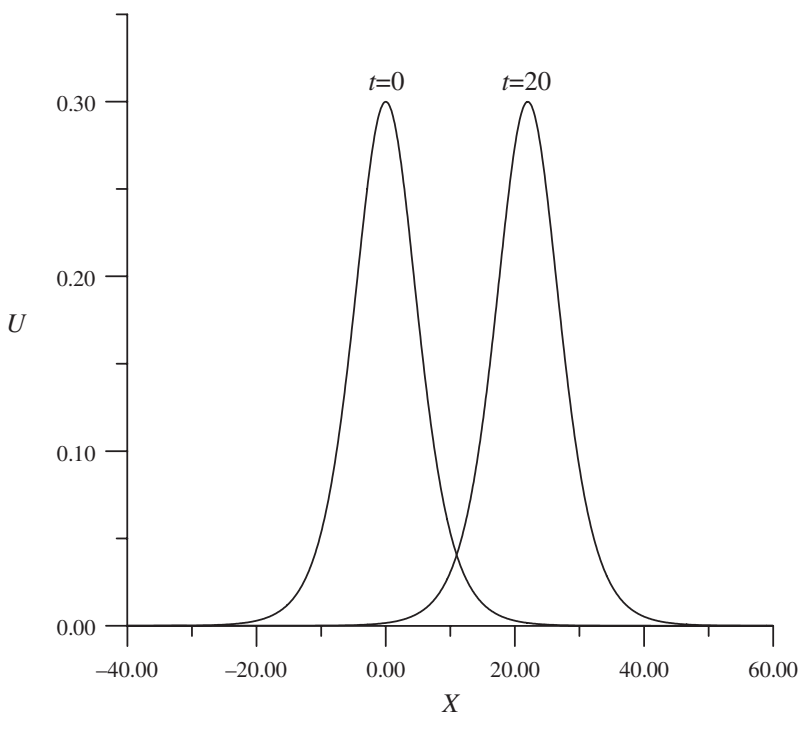

FIGURE 1. Solitary wave solution.

visualization of the solution at time $t=20$ in Figure 1 for the three algorithms. Error (exact solution minus numerical solution) distributions at the same time are depicted in Figures 2-4 to show the errors between the analytical and numerical results over the problem domain. Thus error norms and invariants tell us that the single solitary wave solution of the RLW equation is simulated with little error. A comparison of the quintic B-spline collocation solutions with the variants of both quadratic and cubic B-spline collocation methods and a type of both quintic B-spline Petrov-Galerkin and quadratic B-spline Galerkin finite element methods can be made with error norms exhibited in Table 1. So from the tabulated results, the quadratic B-spline collocation method [6] together with the splitting technique provided one digit less error for the $L_{2}$ error norm than that of the other methods given in Table 1 .

3.2. Interaction of two solitary waves Interaction of two positive solitary waves is studied by using the initial condition

$$
\begin{aligned}
& U(x, 0)=U_{1}+U_{2}, \\
& U_{j}=3 A_{j} \operatorname{sech}^{2}\left(k_{j}\left(x-\tilde{x}_{j}\right)\right), \quad A_{j}=\frac{4 k_{j}^{2}}{1-4 k_{j}^{2}}, \quad j=1,2,
\end{aligned}
$$

together with boundary conditions $U(0, t)=U(120, t)=0, U_{x}(0, t)=U_{x}(120, t)$ $=0$. The parameters $k_{1}=0.4, \widetilde{x}_{1}=15, k_{2}=0.3, \widetilde{x}_{2}=35$ are chosen to coincide with those used in the literature $[1,9,17]$. These parameters provide solitary waves of magnitudes about 5.33333 and 1.68750 and their peak positions are located at $x=15$ and 35. Calculations are carried out with the time step $\Delta t=0.1$ and mesh size $h=0.1$ over the region $0 \leq x \leq 120$. 


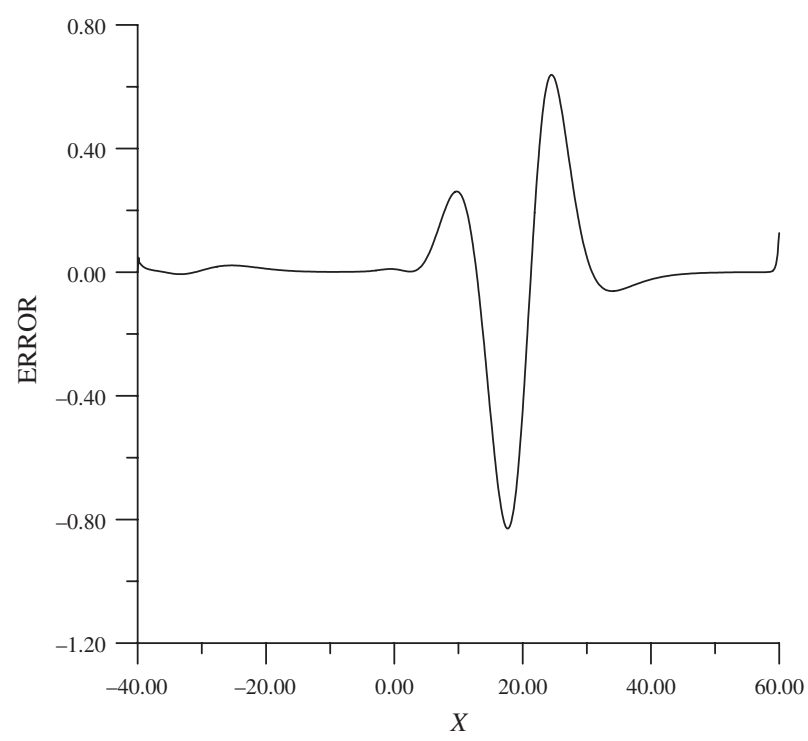

FIGURE 2. Error $\times 10^{4}(\mathrm{QBCM} 1)$.

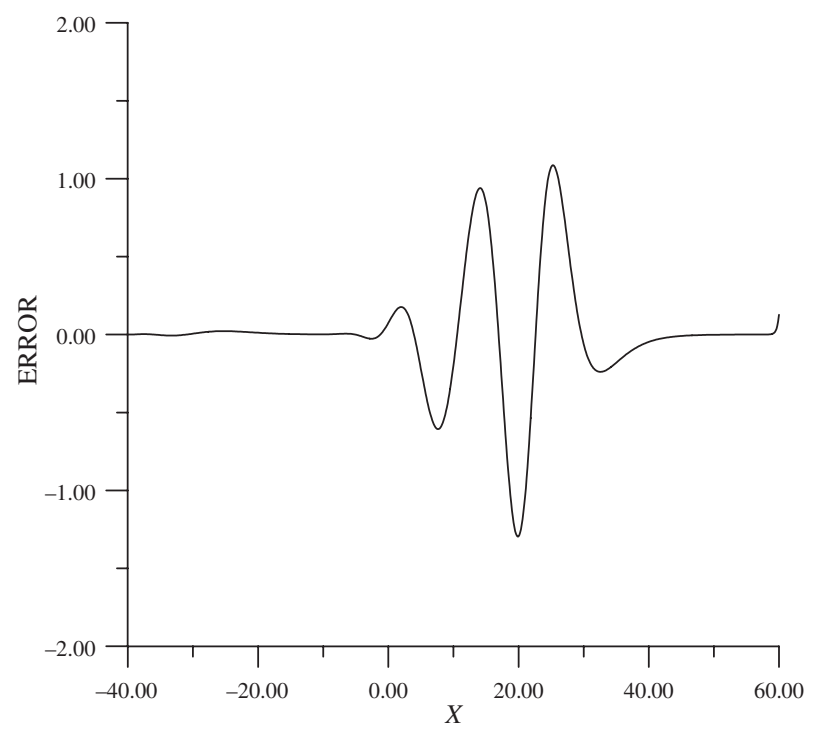

FIGURE 3. Error $\times 10^{4}(\mathrm{QBCM} 2)$. 


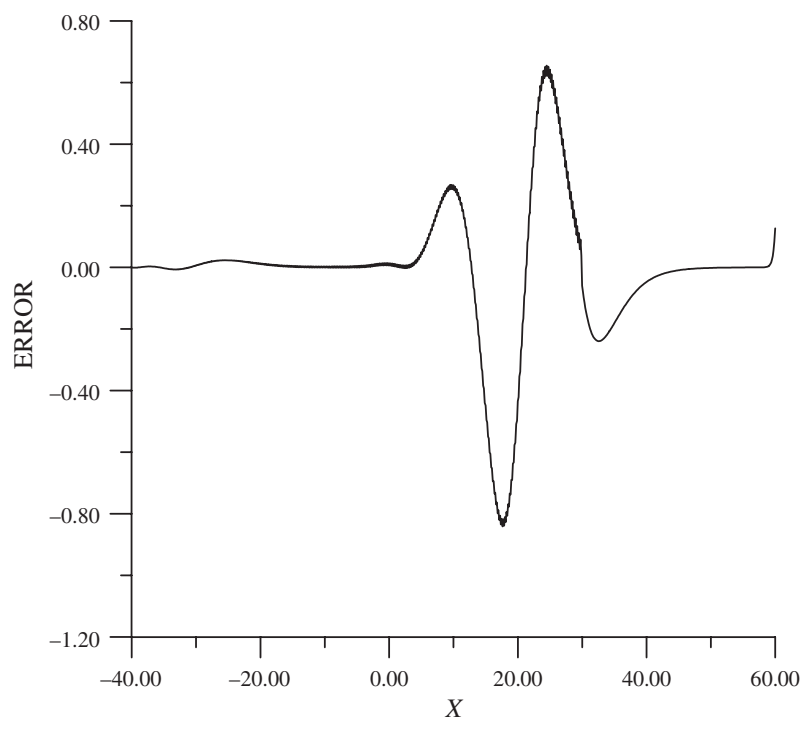

FIGURE 4. Error $\times 10^{4}(\mathrm{QBCM} 3)$.

TABLE 1. Invariants and error norms for a single solitary wave at time $t=20$ : amplitude $=0.3$.

\begin{tabular}{llllll}
\hline$L_{2} \times 10^{4}$ & $L_{\infty} \times 10^{4}$ & \multicolumn{1}{c}{$C_{1}$} & \multicolumn{1}{c}{$C_{2}$} & \multicolumn{1}{c}{$C_{3}$} & \\
\hline 0.0 & 0.0 & 3.9799271 & 0.8104625 & 2.5790075 & $(t=0)$ \\
2.15192 & 0.82951 & 3.9798798 & 0.8104625 & 2.5790075 & (QBCM1) \\
3.56672 & 1.29682 & 3.9798843 & 0.8104625 & 2.5790074 & (QBCM2) \\
2.15192 & 0.82951 & 3.9798798 & 0.8104625 & 2.5790075 & (QBCM3) \\
3.01 & 1.14 & 3.97996 & 0.810276 & 2.57839 & (cub. met. [13]) \\
1.92 & 0.73 & 3.97989 & 0.81046 & 2.57901 & (quad. gal. [21]) \\
2.2050 & 0.8448 & 3.980016 & 0.8104624 & 2.579006 & (CBSCM2 [6]) \\
2.6086 & 1.0299 & 3.979958 & 0.8104596 & 2.578999 & (CBSCM1 [6]) \\
0.4315 & 0.1321 & 3.979890 & 0.8104625 & 2.578999 & (QBSCM [6]) \\
3.7841 & 1.3993 & 3.97995 & 0.81046 & 2.57900 & (cub. met. [20]) \\
2.2 & 0.86 & 3.97989 & 0.810467 & 2.57902 & (quad. gal. $(h=0.1)[12])$ \\
2.38 & 0.87 & 3.97995 & 0.810459 & 2.57900 & (quin. met. $(h=0.2)[11])$ \\
\hline
\end{tabular}

The initial solitary waves with given parameters are shown in Figure 5. These solitary waves are propagated to the right with velocities that depend upon their magnitudes and have reached a stage where the larger wave has passed through the smaller solitary wave and emerged with their original positions. The shapes of both waves are graphed during the interaction period at time $t=15$ and after the interaction at time $t=30$ in Figure 5 for the three schemes. For QBCM1, at time $t=30$, the smaller solitary wave of amplitude 1.68285 , whose peak is located at $x=77.9$, is recorded and the larger solitary wave of amplitude 5.32322 is obtained, the peak position of which is located at $x=100.8$. At the same time, using the second algorithm 


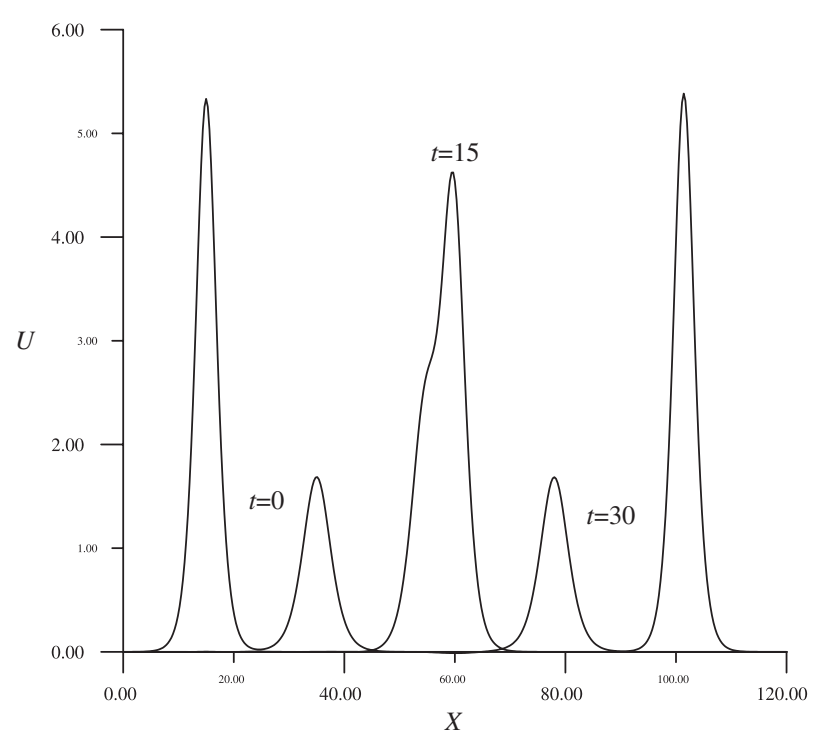

FIGURE 5. Two solitary wave solutions.

QBCM2, we have found the amplitudes of both smaller and larger waves as 1.68370 and 5.327 72, and the $x$-positions of the peaks of the amplitudes are 77.9 and 100.8 respectively. Using the third algorithm QBCM3, we have found the amplitudes of both smaller and larger waves as 1.68285 and 5.32322 , and the $x$-positions of the peaks of the amplitudes are 77.9 and 100.8 respectively. It is found for the schemes QBCM1, QBCM2 and QBCM3 that the absolute difference of larger amplitude between times $t=0$ and $t=30$ is $1.011 \times 10^{-2}, 5.61 \times 10^{-3}, 1.011 \times 10^{-2}$ and that of smaller amplitude is $4.65 \times 10^{-3}, 3.8 \times 10^{-3}, 4.65 \times 10^{-3}$, respectively.

The interaction process can be observed clearly from the graph of the timeamplitude in Figure 6. Thus, the larger solitary wave started to catch up the small solitary wave at about time $t=10$. The interaction time continued to end up at about time $t=20$ and the two solitary waves have regained their original amplitudes after the interaction. In Figures 7-10, we depict the values of the invariants $C_{1}, C_{2}$ and $C_{3}$ against time throughout the simulation for the three algorithms. It can be seen from these figures that, during a run of the algorithms, the conservation laws are changed in the ranges as $37.9164 \leq C_{1} \leq 37.9171,120.5233 \leq C_{2} \leq 120.5263$, $743.4675 \leq C_{3} \leq 744.1017$ for QBCM1, $37.9164 \leq C_{1} \leq 37.9170,120.5233 \leq C_{2}$ $\leq 120.5256,743.6011 \leq C_{3} \leq 744.2264$ for QBCM2, and $37.9165 \leq C_{1} \leq 37.9170$, $120.5233 \leq C_{2} \leq 120.5264,743.4675 \leq C_{3} \leq 744.1017$ for QBCM3.

3.3. Wave undulation We study the undulation from the initial condition

$$
U(x, 0)=0.5 U_{0}\left[1-\tanh \left(\frac{x-x_{c}}{d}\right)\right],
$$




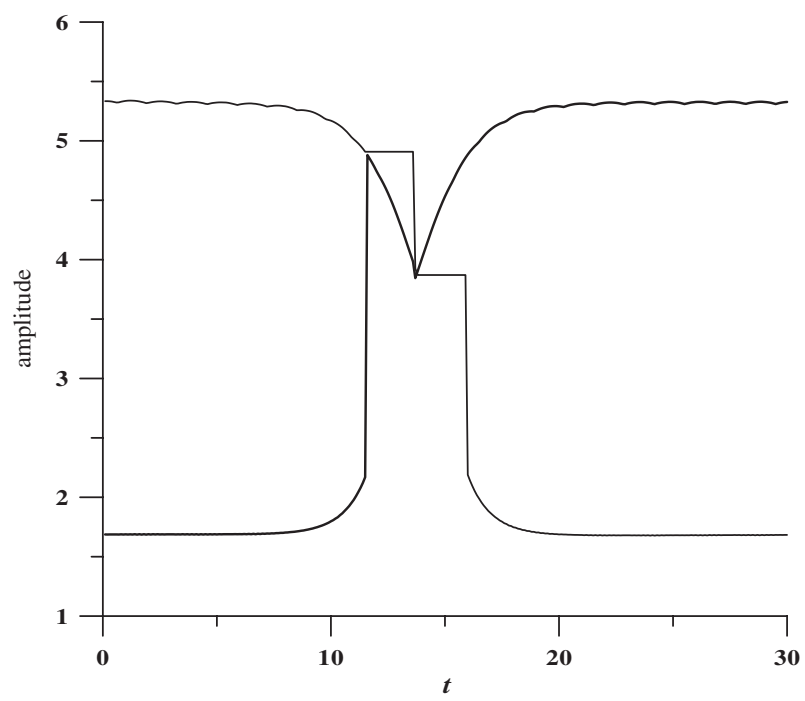

FIGURE 6. Wave amplitudes.

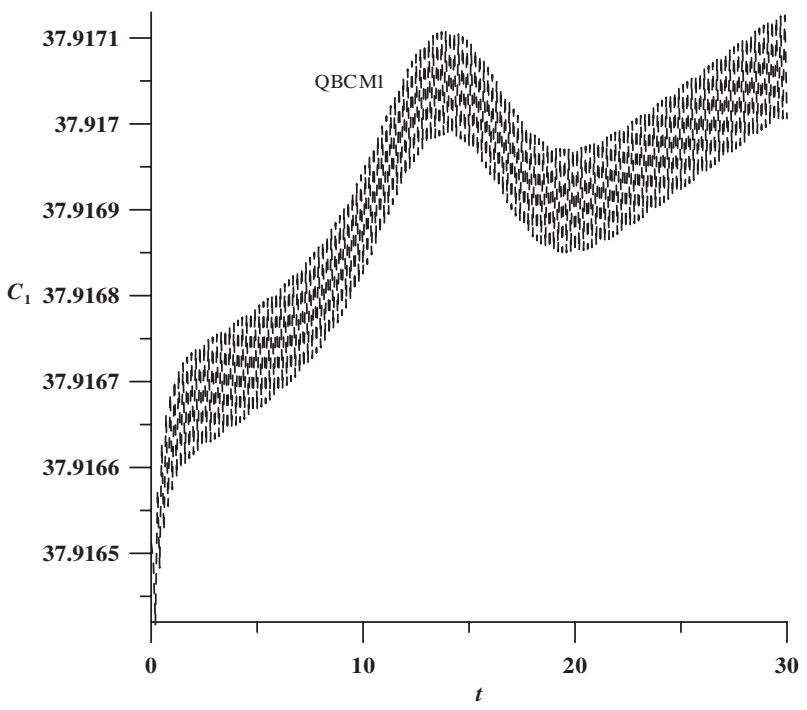

FIGURE 7. $C_{1}$ quantities curves. 


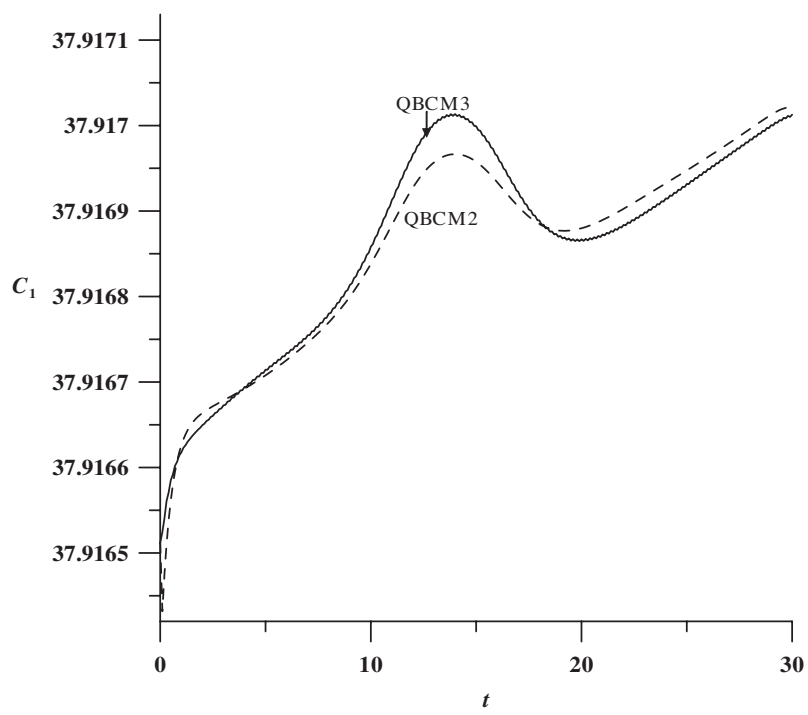

FIGURE 8. $C_{1}$ quantities curves.



Figure 9. $C_{2}$ quantities curves. 




FIGURE 10. $C_{3}$ quantities curves.

and the following boundary conditions are used:

$$
U(a, t)=U_{0}, \quad U(b, t)=U_{x}(a, t)=U_{x}(b, t)=0,
$$

where $U(x, 0)$ denotes the elevation of the water above the equilibrium surface at time $t=0$ and $d$ is the slope between the still water and deeper water. The change in water level of magnitude $U(x, 0)$ is centred on $x=x_{c}$. The parameters $\varepsilon=1.5$, $\mu=0.16666667, U_{0}=0.1, x_{c}=0, a=-36, b=300, h=0.24, \Delta t=0.1, d=2,5$ are taken to make comparison with results of $[2,4,6,8,11,20]$. The results of the algorithms for the RLW equation are obtained up to time $t=250$ with the initial condition (3.7). The initial undulation form has evolved into a steady-state profile moving in the positive direction with more undulations. Views of both numerical solutions and the initial wave are depicted at time $t=250$ for the three schemes in Figures 11 and 12. The amplitudes of the successive four undulations and the positions of their peak values are documented in Table 2. It can be seen from Table 2 that the magnitudes of the amplitudes and the $x$-values of the peak positions of undulations for all of the algorithms presented in this paper are found to be almost the same. The results and graphs of simulations agree with those of published papers $[2,4,6,8,11,20]$.

\section{Conclusion}

The main purpose of this paper has been to compare the numerical findings when the quintic B-spline is used in the collocation method, and achievement of the splitting technique in the numerical methods has been searched. So numerical solutions of the 




FIGURE 11. Results for $d=5$.

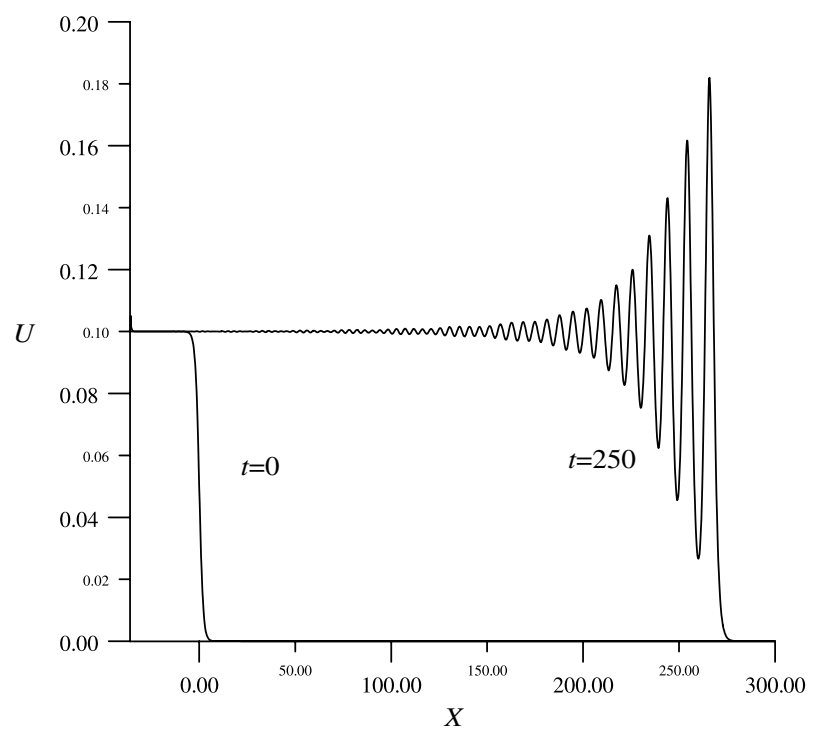

FIGURE 12. Results for $d=2$. 
TABle 2. Positions and amplitudes of the undulations at time $t=250$.

\begin{tabular}{|c|c|c|c|c|}
\hline \multirow{2}{*}{$\overline{\mathrm{QBCM} 1}$} & \multicolumn{2}{|c|}{$d=5$} & \multicolumn{2}{|c|}{$d=2$} \\
\hline & Position & Amplitude & Position & Amplitude \\
\hline Leading undu. & 264.96 & 0.1778726718 & 265.92 & 0.1819824964 \\
\hline Second undu. & 253.92 & 0.1533326019 & 254.16 & 0.1620409707 \\
\hline Third undu. & 244.08 & 0.1324152938 & 244.08 & 0.1445499209 \\
\hline Fourth undu. & 234.96 & 0.1178158182 & 234.72 & 0.1305279787 \\
\hline \multirow[t]{2}{*}{$\overline{\mathrm{QBCM} 2}$} & \multicolumn{2}{|c|}{$d=5$} & \multicolumn{2}{|c|}{$d=2$} \\
\hline & Position & Amplitude & Position & Amplitude \\
\hline Leading undu. & 264.96 & 0.1779099030 & 265.92 & 0.1820383624 \\
\hline Second undu. & 253.92 & 0.1533978682 & 254.16 & 0.1620437130 \\
\hline Third undu. & 244.08 & 0.1324837496 & 244.08 & 0.1446074770 \\
\hline Fourth undu. & 234.96 & 0.1178715349 & 234.72 & 0.1306019286 \\
\hline \multirow[t]{2}{*}{ QBCM3 } & \multicolumn{2}{|c|}{$d=5$} & \multicolumn{2}{|c|}{$d=2$} \\
\hline & Position & Amplitude & Position & Amplitude \\
\hline Leading undu. & 264.96 & 0.1777572725 & 265.68 & 0.1817862439 \\
\hline Second undu. & 253.92 & 0.1529425601 & 254.16 & 0.1617628974 \\
\hline Third undu. & 244.08 & 0.1318675602 & 244.08 & 0.1438498473 \\
\hline Fourth undu. & 234.96 & 0.1172800241 & 234.72 & 0.1296158837 \\
\hline
\end{tabular}

RLW equation have been constructed by using the collocation method with quintic $\mathrm{B}$-splines as interpolation functions. The RLW equation is split both in space and in time. Those coupled systems of differential equations are also solved by way of the quintic B-spline collocation method over uniform finite intervals. Although the three ways of solving the equation produce very close results, the space-splitting process together with numerical methods produce slightly better results than the time-splitting one. The disadvantage is that the space-splitting of the equation is costly due to the high-order matrix system being obtained after the application of the numerical method. We have also observed that the space/time-splitting procedure for the RLW equation does not result in much increase of accuracy in the quintic B-spline collocation method. Consequently, quintic B-spline functions are handy in writing the approximate solutions in the numerical methods if the partial differential equations involve higher-order derivatives.

\section{References}

[1] Kh. O. Abdulloev, I. L. Bogolubsky and V. G. Makhankov, "One more example of inelastic soliton interaction", Phys. Lett. A 56 (1976) 427-428.

[2] P. Avilez-Valente and F. J. Seabra-Santos, "A Petrov-Galerkin finite element scheme for the regularized long wave equation”, Comput. Mech. 34 (2004) 256-270.

[3] T. B. Benjamin, J. L. Bona and J. J. Mahony, "Model equations for long waves in nonlinear dispersive systems", Philos. Trans. R. Soc., Lond., Ser. A 272 (1972) 47-78.

[4] D. Bhardwaj and R. Shankar, "A computational method for regularized long wave equation", Comput. Math. Appl. 40 (2000) 1397-1404.

[5] J. L. Bona and P. J. Bryant, "A mathematical model for long waves generated by wave makers in nonlinear dispersive systems", Proc. Cambridge Philos. Soc. 73 (1973) 391-405. 
[6] İ. Dağ, A. Doğan and B. Saka, "B-spline collocation methods for numerical solutions of the RLW equation", Int. J. Comput. Math. 80 (2003) 743-757.

[7] I. Dağ, B. Saka and A. Boz, "Quintic B-spline Galerkin methods for numerical solutions of the Burgers' equation”, in Proc. Int. Conf. Dynamical Systems and Applications, Antalya, Turkey, 5-10 July 2004 (Altas Conferences Inc.), 295-309.

[8] A. Esen and S. Kutluay, "Application of a lumped Galerkin method to the regularized long wave equation", Appl. Math. Comput. 174 (2006) 833-845.

[9] L. R. T. Gardner and G. A. Gardner, "Solitary wave of the regularized long wave equation", J. Comput. Phys. 91 (1990) 441-459.

[10] G. A. Gardner, L. R. T. Gardner and A. H. A. Ali, "Modelling solitons of the Korteweg-de Vries equation with quintic B-splines", U.C.N.W. Math., Preprint, 1990.

[11] L. R. T. Gardner, G. A. Gardner, F. A. Ayoub and N. K. Amein, "Modelling an undular bore with B-splines", Comput. Methods Appl. Mech. Engrg. 147 (1997) 147-152.

[12] L. R. T. Gardner, G. A. Gardner and İ. Dağ, "A B-spline finite element method for the regularized long wave equation”, Comm. Numer. Methods Engrg. 11 (1995) 59-68.

[13] D. Irk, İ. Dağ and A. Doğan, "Numerical integration of the RLW equation using cubic splines", ANZIAM J. 47 (2005) 131-142.

[14] P. J. Olver, "Euler operators and conservation laws of the BBM equation", Math. Proc. Cambridge Philos. Soc. 85 (1979) 143-159.

[15] D. H. Peregrine, "Calculations of the development of an undular bore", J. Fluid. Mech. 25 (1966) 321-330.

[16] P. M. Prenter, Splines and variational methods (John Wiley \& Sons, New York, 1975).

[17] K. R. Raslan, "A computational method for the regularized long wave (RLW) equation", Appl. Math. Comput. 167 (2005) 1101-1118.

[18] V. Rosenberg, Methods for solution of partial differential equations, Vol. 113 (Elsevier, New York, 1969).

[19] B. Saka, "A finite element method for equal width equation", Appl. Math. Comput. 175 (2006) 730-747.

[20] B. Saka and İ. Dağ, "A collocation method for the numerical solution of the RLW equation using cubic B-spline basis", Arab. J. Sci. Eng. 30 (2005) 39-50.

[21] B. Saka, İ. Dağ and A. Doğan, "Galerkin method for the numerical solution of the RLW equation using quadratic B-splines”, Int. J. Comput. Math. 81 (2004) 727-739.

[22] M. Shashkov, Conservative finite-difference methods on general grids (CRC Press, Boca Raton, FL, 1996).

[23] S. I. Zaki, "A quintic B-spline finite elements scheme for the KdVB equation”, Comput. Methods Appl. Mech. Engrg. 188 (2000) 121-134. 